\title{
Volume growth, curvature decay, and critical metrics
}

\author{
Gang Tian* and Jeff Viaclovsky**
}

\begin{abstract}
We make some improvements to our previous results in [TV05a] and [TV05b]. First, we prove a version of our volume growth theorem which does not require any assumption on the first Betti number. Second, we show that our local regularity theorem only requires a lower volume growth assumption, not a full Sobolev constant bound. As an application of these results, we can weaken the assumptions of several of our theorems in [TV05a] and [TV05b].
\end{abstract}

Mathematics Subject Classification (2000). Anti-self-dual metrics, ALE spaces, curvature decay, $\varepsilon$-regularity, orbifold compactness, volume growth.

Keywords. Primary 53C21; Secondary 58D27, 58E11.

\section{Introduction}

Riemannian spaces with quadratic curvature decay have been widely studied in the literature, see for example [Abr85], [Abr87], [AG90], [BKN89], [Kas88], [Kas89], [GPZ94], [Gro81], [Zhu94]. All of these works assume that the curvature decay is strictly better than quadratic in the sense that

$$
|\mathrm{Rm}|=O\left(r^{-(2+\delta)}\right), \quad \text { as } r \rightarrow \infty,
$$

for some $\delta>0$, where $r(x)=d(p, x)$ is the distance to a basepoint $p$, or the weaker assumption that

$$
\mathrm{Rm} \geq-\frac{k(r)}{r^{2}},
$$

(meaning all of the sectional curvatures are bounded below accordingly), and the function $k(r)$ satisfies

$$
\int_{1}^{\infty} \frac{k(r)}{r} d r<\infty
$$

\footnotetext{
*The research of the first author was partially supported by NSF Grant DMS-0302744.

${ }^{* *}$ The research of the second author was partially supported by NSF Grant DMS-0503506.
} 
Spaces satisfying such a curvature decay condition are said to have asymptotically nonnegative curvature. We remark that by standard comparison theory, (1.2) and (1.3) imply an upper volume growth estimate,

$$
\operatorname{Vol}(B(p, r)) \leq V_{0} r^{n},
$$

for some constant $V_{0}$. Moreover, only a lower bound on the Ricci curvature is needed for this upper volume growth estimate [Zhu94].

It has been understood that such a volume estimate is the core of extending orbifold-compactness theorems for Einstein metrics to metrics satisfying more general equations, such as anti-self-dual or harmonic curvature. To this end, in our investigation of critical metrics in [TV05a], [TV05b], and in the work of [And05], spaces arise with curvature decay as in (1.2), but the function $k(r)$ only satisfies $k(r) \rightarrow 0$ as $r \rightarrow \infty$, and standard comparison arguments do not apply. In [TV05a] we proved an upper volume growth estimate in this case, but our proof required finiteness of the first Betti number to rule out the presence of so-called "bad" annuli. In this paper, we show that adding the condition (1.4) below, eliminates this pathology. For $M$ non-compact, $C_{S}$ is defined to be the best constant so that

$$
\|f\|_{L^{\frac{2 n}{n-2}}} \leq C_{S}\|\nabla f\|_{L^{2}}
$$

for all $f \in C^{0,1}(M)$ with compact support. Let Ric - denote the negative part of the Ricci tensor.

Theorem 1.1. Let $(M, g)$ be a complete, noncompact, n-dimensional Riemannian manifold with base point $p$. Assume that

$$
C_{S}<\infty
$$

and that

$$
\sup _{S(r)}\left|\operatorname{Rm}_{g}\right|=o\left(r^{-2}\right)
$$

as $r \rightarrow \infty$, where $S(r)$ denotes the sphere of radius $r$ centered at $p$. If

$$
\int_{M} \mid \text { Ric }\left._{-}\right|^{\frac{n}{2}} d V_{g}<\Lambda,
$$

for some constant $\Lambda \in \mathbb{R}$, then $(M, g)$ has finitely many ends, and there exists a constant $C_{2}$ (depending on $g$ ) so that

$$
\operatorname{Vol}(B(p, r)) \leq C_{2} r^{n} .
$$

Furthermore, each end is ALE of order 0. 
We have another generalization of our previous results. Consider any system of the type

$$
\Delta \mathrm{Ric}=\mathrm{Rm} * \mathrm{Ric},
$$

where the right hand side is shorthand notation for a linear combination of terms of the form $A_{i j k l} B_{j l}$, where $A_{i j k l}$ depends on the full curvature tensor, and $B_{j l}$ depends only on the Ricci tensor. We call any metric satisfying a system of the form (1.5) a critical metric. In [TV05a, Theorem 3.1], we proved an $\varepsilon$-regularity theorem in dimension four which depended on the Sobolev constant. Here we relax this condition and require only a lower volume growth assumption:

Theorem 1.2. Let $(M, g)$ be a four-dimensional Riemannian manifold. Assume that (1.5) is satisfied, let $r<\operatorname{diam}(M) / 2, B(p, r)$ be a geodesic ball around the point $p$, and $k \geq 0$. If there exists a constant $V_{0}>0$ so that

$$
\operatorname{Vol}(B(q, s)) \geq V_{0} s^{4}
$$

for all $q \in B(p, r / 2)$ and $s \leq r / 2$, then there exist constants $\varepsilon_{0}, C_{k}$ (depending upon $\left.V_{0}\right)$ so that if

$$
\|\mathrm{Rm}\|_{L^{2}(B(p, r))}=\left\{\int_{B(p, r)}|\mathrm{Rm}|^{2} d V_{g}\right\}^{1 / 2} \leq \varepsilon_{0},
$$

then

$$
\sup _{B(p, r / 2)}\left|\nabla^{k} \mathrm{Rm}\right| \leq \frac{C_{k}}{r^{2+k}}\left\{\int_{B(p, r)}|\mathrm{Rm}|^{2} d V_{g}\right\}^{1 / 2} \leq \frac{C_{k} \varepsilon_{0}}{r^{2+k}} .
$$

Theorems 1.1 and 1.2 are the main results in this paper. A consequence is that we can (i) remove the Betti number assumption from our volume growth theorem from [TV05b, Theorem 1.2], or (ii) we can relax the Sobolev constant assumption to only an assumption on lower volume growth.

Recall that a metric $g$ is called anti-self-dual if $W_{g}^{+} \equiv 0$, and self-dual if $W_{g}^{-} \equiv 0$. As in [TV05b], we specialize to the class of

(a) self-dual or anti-self-dual metrics with constant scalar curvature,

(b) metrics with harmonic curvature $(\delta \mathrm{Rm} \equiv 0)$,

(c) Kähler metrics with constant scalar curvature.

We have the following notion of local Sobolev constant. For $p \in M$, and $r>0$, we define $C_{S}(p, r)$ to be the best constant such that

$$
\|f\|_{L^{4}} \leq C_{S}(p, r)\|\nabla f\|_{L^{2}}
$$


for all $f \in C^{0,1}$ with compact support in $B(p, r)$, and define

$$
C_{S}(r)=\sup _{p \in M} C_{S}(p, r) .
$$

Let $b_{1}(M)$ denote the first Betti number of $M$.

Theorem 1.3. Let $(M, g)$ be a metric of class (a), (b), or (c) on a smooth, complete four-dimensional manifold $M$ satisfying

$$
\int_{M}\left|\operatorname{Rm}_{g}\right|^{2} d V_{g} \leq \Lambda
$$

for some constant $\Lambda$.

Assume that

$$
\begin{aligned}
\operatorname{Vol}(B(q, s)) & \geq V_{0} s^{4}, \quad \text { for all } q \in M, \text { and } s \leq \operatorname{diam}(M) / 2, \\
b_{1}(M) & <B_{1},
\end{aligned}
$$

where $V_{0}, B_{1}$ are constants. Then there exists a constant $V_{1}$, depending only upon $V_{0}, \Lambda, B_{1}$, such that $\operatorname{Vol}(B(p, r)) \leq V_{1} \cdot r^{4}$, for all $p \in M$ and $r>0$.

Assume instead that

$$
C_{S}(r)<C_{1}, \quad \text { for } r<\operatorname{diam}(M) / 2,
$$

where $C_{1}$ is a constant. Then there exists a constant $V_{2}$, depending only upon $C_{1}, \Lambda$, such that $\operatorname{Vol}(B(p, r)) \leq V_{2} \cdot r^{4}$, for all $p \in M$ and $r>0$.

We have the following improvement of our compactness theorem [TV05b, Theorem 1.3] (we refer to that work for the definition of a multi-fold):

Theorem 1.4. Let $\left(M_{i}, g_{i}\right)$ be a sequence of unit-volume metrics of class (a), (b) or (c) on smooth, closed four-dimensional manifolds $M_{i}$ satisfying

$$
\int_{M_{i}}\left|\mathrm{Rm}_{g_{i}}\right|^{2} d V_{g_{i}} \leq \Lambda
$$

where $\Lambda$ is a constant.

Assume that

$$
\begin{aligned}
\operatorname{Vol}(B(q, s)) & \geq V_{0} s^{4}, \quad \text { for all } q \in M, \text { and } s \leq \operatorname{diam}(M) / 2, \\
b_{1}\left(M_{i}\right) & <B_{1},
\end{aligned}
$$

where $C_{1}, \Lambda, B_{1}$ are constants. Then a subsequence converges to a limit metric space $\left(M_{\infty}, g_{\infty}\right)$ which is a compact, connected, critical Riemannian multi-fold. The convergence is smooth away from a finite singular set. 
If we assume instead that

$$
C_{S g_{i}}(r)<C_{1}, \quad \text { for } r<\operatorname{diam}(M) / 2,
$$

then the same conclusion is true.

Remark. The first Betti number assumption from [TV05a, Theorem 1.1] (which allows non-compact limits) can similarly be removed, and the Sobolev constant assumption relaxed to lower volume growth.

This type of uniform volume estimate, compactness theorem, and related bubbling phenomena follows in a standard fashion from an $\varepsilon$-regularity theorem and a volume growth estimate of the type in Theorem 1.1 and [TV05a, Theorem 1.1]. This passage was first carried out for Einstein metrics [And89], [BKN89], [Tia90], [AC91], [Ban90a], [Ban90b], [Nak92] and extends to more general cases with careful attention to the number of components of geodesic spheres [TV05b], [And05], [CQY07]. In Section 4, we discuss the main technical details of this step under the weaker assumptions in Theorem 1.3 and 1.4.

Let $R_{g}$ denote the scalar curvature of the metric $g$. Using the above in case (a), we note the following special corollary.

Corollary 1.5. Let $\left(M, g_{i}\right)$ be a sequence of unit volume constant scalar curvature anti-self-dual metrics on a fixed closed 4-manifold M. Assume that

$$
\begin{aligned}
\left|R_{g_{i}}\right| & <C, \\
\operatorname{Vol}(B(q, s)) & \geq V_{0} s^{4}, \quad \text { for all } q \in M, \text { and } s \leq \operatorname{diam}(M) / 2,
\end{aligned}
$$

where $C, V_{0}$ are constants. Then a subsequence converges to a limit space $\left(M_{\infty}, g_{\infty}\right)$ which is a compact, connected, anti-self-dual Riemannian multi-fold.

Acknowledgements. The authors would like to thank Gilles Carron, John Lott and Joao Santos for enlightening discussions. We also thank Xiuxiong Chen, Edward Fan and Brian Weber for insightful questions and comments on our previous work.

\section{Proof of Theorem 1.1}

The space of $L^{2}$-harmonic $k$-forms $\mathscr{H}^{k}(M)$ is defined to be those $\omega \in \Lambda^{k}\left(T^{*} M\right)$ satisfying $\Delta \omega=0$, and $\omega \in L^{2}(M)$. It is well-known that $\mathscr{H}^{k}(M) \simeq \bar{H}_{(2)}^{k}(M)$, the reduced $L^{2}$-cohomology, see [Car99]. We next quote the following finiteness theorem. 
Theorem 2.1 (Carron, [Car98], [Car99]). Let $(M, g)$ be a complete Riemannian manifold satisfying for $p>2$ the Sobolev inequality

$$
\mu_{p}(M)\left(\int_{M}|u|^{\frac{2 p}{p-2}}(x) d V_{g}\right)^{1-\frac{2}{p}} \leq \int_{M}|d u|^{2}(x) d V_{g}, \quad \text { for all } u \in C_{c p t}^{\infty}(M) .
$$

If the negative part of the Ricci satisfies

$$
\int_{M} \mid \text { Ric }\left._{-}\right|^{p / 2} d V_{g}<\infty
$$

then $\mathscr{H}^{1}(M)$ is finite dimensional. If the full Riemannian tensor curvature satisfies

$$
\int_{M}|\mathrm{Rm}|^{p / 2} d V_{g}<\infty
$$

then $\mathscr{H}^{k}(M)$ is finite dimensional for each $1 \leq k \leq n$.

Remark. For convenience of the reader, we give an indication of the proof in [Car99]. A crucial estimate is that the Sobolev constant bound yields an estimate on the heat kernel: there exists a constant $C$ such that for all $x \in M$, and any $t>0$,

$$
k(t, x, x) \leq C t^{-n / 2} .
$$

It is then shown that $\mathscr{H}^{k}(M)$ is finite dimensional, using the Cwickel-Lieb-Rosenbljum estimate adapted to the Riemannian setting. Similar results were also obtained in [BB90]. The Weitzenbock formula for a 1-form is

$$
\nabla^{*} \nabla=\Delta_{H}+\text { Ric, }
$$

so for the case of harmonic 1-forms, only the Ricci assumption (2.2) is necessary. For $k>1$, the Weitzenbock formula depends upon the full curvature tensor, which is why the full curvature assumption (2.3) is required. We note that this method also gives an explicit estimate on the dimension of $\mathscr{H}^{k}(M)$ in terms of the Sobolev constant $\mu_{p}(M)$, and the $L^{p / 2}$ curvature integral.

We recall a definition.

Definition 2.2. We say a component $A_{0}\left(r_{1}, r_{2}\right)$ of an annulus $A\left(r_{1}, r_{2}\right)=\{q \in M \mid$ $\left.r_{1}<d(p, q)<r_{2}\right\}$ is bad if $S\left(r_{1}\right) \cap \overline{A_{0}\left(r_{1}, r_{2}\right)}$ has more than one component, where $S\left(r_{1}\right)$ is the sphere of radius $r_{1}$ centered at $p$.

For an annulus $A_{0}\left(r_{1}, r_{2}\right)$, we call a component of $S\left(r_{1}\right) \cap \overline{A_{0}\left(r_{1}, r_{2}\right)}$ an inner sphere. Note that this may have several components - indeed, this is one of the main subtleties in proving the volume growth estimate in Theorem 1.1. 
Theorem 2.3. Under the assumption in Theorem 1.1, there exists a constant $N_{0}$ (depending upon $C_{S}$ and $\Lambda$ ) such that if $A_{1}, A_{2}, \ldots, A_{N}$ is a collection of disjoint connected bad annuli, then $N<N_{0}$.

Proof. From the assumptions in Theorem 1.1, the estimate (2.1) is satisfied for $p=n$, therefore Theorem 2.1 says that $\mathscr{H}^{1}$ is finite dimensional. Letting $H^{1}(M)$ and $H_{c}^{1}(M)$ denote the first cohomology and first cohomology with compact support, respectively, this implies that

$$
\operatorname{Image}\left(H_{c}^{1}(M) \rightarrow H^{1}(M)\right)
$$

has finite dimension, since the space in (2.4) injects into $\mathscr{H}^{1}$ (see [And88]). The rest of the argument just uses the finiteness of (2.4). Let $A_{i}=A\left(r_{i}, s_{i}\right)$. Without loss of generality, let us assume that the sequence of radii $r_{i}$ is non-decreasing. Since $A_{i}$ is bad, the inner sphere $S\left(r_{i}\right) \cap A_{i}$ has $N_{i}>1$ components, call them $\mathcal{C}_{i, j}, j=1, \ldots, N_{i}$. Take any two components, say $\mathcal{C}_{i, 1}$ and $\mathcal{C}_{i, 2}$, and let $p_{i, j}$ be any point of $\mathcal{C}_{i, j}$. For fixed $i$, let $\gamma_{i, 2,1}(t)$ be a curve in $A_{i}$ connecting $p_{i, 2}$ with $p_{i, 1}$. We can always find such a curve since, by assumption, $A_{i}$ is connected. We can also find a curve $\alpha_{i, 1,2}(t)$ connecting $p_{i, 1}$ with $p_{i, 2}$, with image in $B\left(p, r_{i}\right)$. Joining these curves, we find a closed loop $\beta_{i}=\gamma_{i, 2,1} \# \alpha_{i, 1,2}$ based at $p_{i, 1}$

Next, we find a function $f_{i}$ defined on $\bar{A}_{i}$ such that $f_{i}$ is supported in a neighborhood of $\mathcal{C}_{i, 1}$, with $f_{i}=1$ on smaller neighborhood of $\mathcal{C}_{i, 1}$, and $f_{i}=0$ in a neighborhood of all other components $\mathcal{C}_{i, j}$. Then the 1 -form $\alpha_{i}=d f_{i}$ clearly has an extension to a smooth 1-form on $M$, which is closed (but not exact), and is supported on $A_{i}$. We claim that

$$
\int_{\beta_{i}} \alpha_{i}=1
$$

To see this, since $\alpha_{i}$ is supported on $A_{i}$,

$$
\int_{\beta_{i}} \alpha_{i}=\int_{\gamma_{i, 2,1}(t)} \alpha_{i}=\int_{\gamma_{i, 2,1}(t)} d f=f\left(p_{i, 1}\right)-f\left(p_{i, 2}\right)=1 .
$$

Furthermore,

$$
\int_{\beta_{i}} \alpha_{j}=0, \quad i<j
$$

This is true since we have assumed the annuli are indexed by increasing radius, the $\alpha_{j}$ forms are supported either on a different component, or on an annulus which is further out. We have shown that the $\alpha_{i}$ define non-zero independent cohomology classes in Image $\left(H_{c}^{1}(M) \rightarrow H^{1}(M)\right)$, and we therefore have

$$
N_{0} \leq \operatorname{dim}\left\{\operatorname{Image}\left(H_{c}^{1}(M) \rightarrow H^{1}(M)\right)\right\} .
$$


Remark. We thank Gilles Carron for providing the above argument, which was much simpler than our original proof. Note also that the finiteness of the dimension of (2.4) will be automatically satisfied if either $H^{1}(M), H_{c}^{1}(M)$, or $\mathscr{H}^{1}(M)$ is finite dimensional.

We quote the following theorem from our previous work.

Theorem 2.4 ([TV05b, Theorem 5.2]). Let $(M, g)$ be a complete, non-compact, n-dimensional Riemannian orbifold (with finitely many singular points) with base point $p$. Assume that there exists a constant $C_{1}>0$ so that

$$
\operatorname{Vol}(B(q, s)) \geq C_{1} s^{n},
$$

for any $q \in M$, and all $s \geq 0$. Assume furthermore that as $r \rightarrow \infty$,

$$
\sup _{S(r)}\left|\operatorname{Rm}_{g}\right|=o\left(r^{-2}\right),
$$

where $S(r)$ denotes the sphere of radius $r$ centered at $p$. If $(M, g)$ contains only finitely many disjoint bad annuli, then $(M, g)$ has finitely many ends, and there exists a constant $C_{2}$ so that

$$
\operatorname{Vol}(B(p, r)) \leq C_{2} r^{n},
$$

Furthermore, each end is ALE of order 0.

Theorem 1.1 follows from the above, since we have shown there are only finitely many bad annuli, and noting that the lower volume growth estimate is implied by the Sobolev constant bound (see [TV05a, Lemma 6.1]). Note also that there is an explicit bound on the number of ends in terms of $C_{S}$ and $\Lambda$, see [Car98, Theorem 3.3].

Theorem 2.5. Theorem 1.1 is valid if $(M, g)$ is assumed to be a smooth orbifold with finitely many singular points.

Proof. It can be verified that Carron's arguments are valid for smooth orbifolds, and since Theorem 2.4 is also valid for orbifolds, the proof is identical to the smooth case. Alternatively, instead of using Carron's result for orbifolds, one can argue, albeit non-effectively, as follows. Take a smoothing of $(M, g)$ by cutting out a small ball about each orbifold singularity and gluing in any smooth metric. This can be done because each boundary $S^{3} / \Gamma$ certainly bounds some smooth manifold. We now have a smooth manifold $(\tilde{M}, \tilde{g})$ which is isometric to $(M, g)$ outside of a large ball $B(p, R)$. The manifold $(\tilde{M}, \tilde{g})$ has the same asymptotic behaviour as the original $(M, g)$, so all of the assumptions of Theorem 1.1 are satisfied by $(\tilde{M}, \tilde{g})$. Applying Theorem 1.1, we obtain an upper volume estimate for balls in $(\tilde{M}, \tilde{g})$, which clearly implies an upper volume estimate for the original $(M, g)$, since the asymptotics are the same. 


\section{Sobolev constant and local regularity}

Recall from the introduction that we have the following notion of local Sobolev constant. For $p \in M$, and $r>0$, we define $C_{S}(p, r)$ to be the best constant such that

$$
\|f\|_{L^{4}} \leq C_{S}(p, r)\|\nabla f\|_{L^{2}}
$$

for all $f \in C^{0,1}$ with compact support in $B(p, r)$. Clearly

$$
C_{S}(p, r) \text { is an increasing function of } r,
$$

and

$$
\lim _{r \rightarrow 0} C_{S}(p, r)=C_{E},
$$

where $C_{E}$ is the best constant for the Euclidean Sobolev inequality.

Proposition 3.1. Assume that (1.5) is satisfied, and let $B\left(p_{0}, 2 r\right)$ be a geodesic ball around the point $p_{0}$. Assume there exists a constant $V_{0}>0$ so that

$$
\operatorname{Vol}(B(q, s)) \geq V_{0} s^{4}
$$

for all $q \in B\left(p_{0}, r\right)$, and $s \leq r$.

Then there exists a constant $\varepsilon_{0}$ (depending upon $V_{0}$ ) so that if

$$
\|\mathrm{Rm}\|_{L^{2}\left(B\left(p_{0}, 2 r\right)\right)}=\left\{\int_{B\left(p_{0}, 2 r\right)}|\mathrm{Rm}|^{2} d V_{g}\right\}^{1 / 2} \leq \varepsilon_{0},
$$

then

$$
C_{S}\left(p_{0}, r / 2\right) \leq C V_{0}^{-1 / 4}
$$

and $C$ does not depend on $V_{0}$.

Remark. We conjecture that for (1.5), $\varepsilon_{0}$ can moreover be taken independent of $V_{0}$ and assumption (3.2) is not necessary. This was recently proved for Einstein metrics by Cheeger-Tian [CT06]. In fact, in [CT06, Section 11] this was already conjectured to hold for anti-self-dual metrics and Kähler metrics with constant scalar curvature.

Proof. Without loss of generality, rescale so that $r=1$. The proof goes by contradiction. Assume we have a sequence of critical metrics $g_{i}, i=i, \ldots, \infty$, and a sequence $\varepsilon_{i} \rightarrow 0$ as $i \rightarrow \infty$, with

$$
\|\operatorname{Rm}\|_{L^{2}\left(B\left(p_{0}, 2\right)\right)} \leq \varepsilon_{i},
$$


and that

$$
C_{S}\left(p_{0}, 1 / 2\right)>C V_{0}^{-1 / 4}
$$

(we will choose $C$ later).

We first choose a "nice" ball, one for which the Sobolev constant is controlled in the ball, and also for nearby points. The following lemma is for a fixed metric.

Lemma 3.2. There exist a point $p_{\infty} \in B(p, 1)$ and a radius $0<r_{\infty} \leq 1 / 2$ such that $C_{S}\left(p_{\infty}, r_{\infty}\right)>C V_{0}^{-1 / 4}$, and $C_{S}\left(p, r_{\infty} / 2\right) \leq C V_{0}^{-1 / 4}$ for all $p \in B\left(p_{\infty}, r_{\infty}\right)$.

Proof. From assumption, we have $C_{S}\left(p_{0}, 1 / 2\right)>C V_{0}^{-1 / 4}$. If $C_{S}(p, 1 / 4) \leq C V_{0}^{-1 / 4}$ for all $p \in B\left(p_{0}, 1 / 2\right)$, then let $B=B\left(p_{0}, 1 / 2\right)$. Otherwise, there exists a point $p_{1} \in B\left(p_{0}, 1 / 2\right)$ with $C_{S}\left(p_{1}, 1 / 4\right)>C V_{0}^{-1 / 4}$. If $C_{S}(p, 1 / 8) \leq C V_{0}^{-1 / 4}$, for all $p \in B\left(p_{1}, 1 / 4\right)$, then we let $B=B\left(p_{1}, 1 / 4\right)$. We continue inductively, assume we have chosen $p_{i-1}$ with $p_{i-1} \in B\left(p_{i-2}, 2^{-i+1}\right)$ and $C_{S}\left(p_{i-1}, 2^{-i}\right)>$ $C V_{0}^{-1 / 4}$. If $C_{S}\left(p, 2^{-i}\right) \leq C V_{0}^{-1 / 4}$ for all $p \in B\left(p_{i-1}, 2^{-i}\right)$ then we stop, and let $B=B\left(p_{i-1}, 2^{-i}\right)$. Otherwise, there exists a point $p_{i} \in B\left(p_{i-1}, 2^{-i}\right)$ and $C_{S}\left(p_{i}, 2^{-i-1}\right)>C V_{0}^{-1 / 4}$.

We claim this procedure must stop in finitely many steps. We have

$$
\begin{aligned}
d\left(p_{0}, p_{i}\right) & \leq d\left(p_{0}, p_{1}\right)+\sum_{j=1}^{i-1} d\left(p_{i}, p_{i+1}\right) \\
& <1 / 2+\sum_{j=1}^{i-1} 2^{-j-1} \\
& <1 / 2+1 / 2=1 .
\end{aligned}
$$

The sequence of points $\left\{p_{i}\right\}$ are therefore all contained in the unit ball $B(p, 1)$. If the process did not stop, then there would exists a limit point $q$. Our first restriction on $C$ is that $C V_{0}^{-1 / 4}>C_{E}$. But from the choices, we would clearly have $\lim _{r \rightarrow 0} C_{S}(q, r)>$ $C_{E}$, which contradicts (3.1).

Now we apply the lemma to each metric $g_{i}$, to find points $p_{i} \in B(p, 1)$ (ball in the $g_{i}$ metric) and $r_{i}<1 / 2$, with $C_{S}\left(p_{i}, r_{i}\right)>C V_{0}^{-1 / 4}$, and $C_{S}\left(p, r_{i} / 2\right) \leq C V_{0}^{-1 / 4}$ for all $p \in B\left(p_{i}, r_{i}\right)$ (Sobolev constant with respect to the $g_{i}$ metric).

Now we rescale to make $r_{i}$ unit size, that is, define $\tilde{g}_{i}=r_{i}^{-2} g_{i}$. and consider the sequence of balls $\tilde{B}\left(p_{i}, 2\right)$. By scale invariance, we have

$$
\|\mathrm{Rm}\|_{L^{2}\left(\tilde{B}\left(p_{i}, 2\right)\right)} \leq \varepsilon_{i}
$$


By our previous $\varepsilon$-regularity theorem [TV05a, Theorem 3.1], the curvature and all of its derivatives are uniformly bounded in $B\left(p_{i}, 3 / 2\right)$

$$
\left|\nabla^{k} \mathrm{Rm}\right| \leq C_{k} \varepsilon_{i}
$$

where $C_{k}$ depends upon $k$ and $C V_{0}^{-1 / 4}$. Since $\varepsilon_{i} \rightarrow 0$, we use the theorem of CheegerGromov to extract a flat limit space, $B\left(p_{\infty}, 3 / 2\right)$, with smooth convergence to the limit. From the assumption $\operatorname{Vol}(B(p, r)) \geq V_{0} r^{n}$, and since the limit space is flat, we therefore get a bound on the Sobolev constant of the limit space, $C_{S} \leq C_{1} V_{0}^{-1 / 4}$. Choosing $C>C_{1}$, we arrive at a contradiction.

Theorem 1.2 then follows from Proposition 3.1 and [TV05a, Theorem 3.1].

\section{Main volume estimate}

In this section, we discuss the proof of Theorems 1.3 and 1.4, and Corollary 1.5. We first prove Theorem 1.3.

Proof of Theorem 1.3. The proof is based on the argument from [TV05b], with some modifications. First, let us assume that the volume growth estimate from Theorem 1.3 holds for $r \leq r_{0}$, where $r_{0}$ is some fixed scale. That is, let us assume that

$$
\operatorname{Vol}\left(B_{g_{i}}(p, r)\right) \leq V r^{4}
$$

for all $p \in M_{i}$, and all $r \leq r_{0}$.

From the $\varepsilon$-regularity Theorem $1.2,\left(M_{j}, g_{j}, p_{j}\right)$ converges to a limiting multi-fold $\left(M_{\infty}, g_{\infty}, p_{\infty}\right)$ for some subsequence $\{j\} \subset\{i\}$, with finitely many $C^{0}$-multi-fold singular points (recall that a $C^{0}$-multi-fold point means that for each cone at a singular point, the metric has a continuous extension to the universal cover of the punctured cone). The argument for this is the same as in [TV05b].

Proposition 4.1. The singular points are smooth orbifold points. That is, if $x$ is a singular point, then for some $\delta>0$, the universal cover of $B(x, \delta) \backslash\{x\}$ is diffeomorphic to a punctured ball $B^{4} \backslash\{0\}$ in $\mathbb{R}^{4}$, and the lift of $g$, after diffeomorphism, extends to a smooth critical metric $\tilde{g}$ on $B^{4}$.

Proof. In the case we assume a bound on the Sobolev constant (but no Betti number bound), this follows directly from [TV05b, Theorem 6.4]. In the case where we only assume a lower volume growth bound, we claim that for any singular point $p$, there exists a constant $C_{s}$ so that

$$
\|u\|_{L^{4}(B(p, \varepsilon))} \leq C_{s}\|\nabla u\|_{L^{2}(B(p, \varepsilon))}, \quad u \in C_{c p t}^{0,1}(B(p, \varepsilon)) .
$$


Indeed, in a neighborhood of a singular point, a $C^{0}$-orbifold is just a $C^{0}$-perturbation of a flat cone, so clearly it satisfies a Sobolev inequality. The result then follows again directly from [TV05b, Theorem 6.4].

Proposition 4.2. If (4.1) is satisfied, and $\left(M_{j}, g_{j}, p_{j}\right)$ converges to a limiting multifold $\left(M_{\infty}, g_{\infty}, p_{\infty}\right)$, as $j \rightarrow \infty$, then there is a bound on the number of cones at a singular point of convergence, depending only upon $\Lambda, V_{0}$, and $B_{1}$ in the first case, and depending only upon $\Lambda$ and $C_{S}(r)$ in the second case. The bound does not depend upon the constant $V$ in (4.1).

Proof. We consider the first case. At any limiting multi-fold point $p_{\infty}^{\prime}$, for $\delta>0$ small, we look at the balls $U_{j}=B\left(p_{j}^{\prime}, \delta\right) \subset M_{j}$ where $p_{j}^{\prime} \rightarrow p_{\infty}^{\prime}$ as $j \rightarrow \infty$. These are manifolds with boundary components spherical space forms, with the metrics arbitrarily close to the limiting multi-fold cone metric. We apply the Gauss-Bonnet Theorem to conclude

$$
\chi\left(U_{j}\right) \sim \int_{U_{j}} \mathrm{Rm} * \mathrm{Rm}+\sum_{k} \frac{1}{\left|\Gamma_{k}\right|} .
$$

where $\mathrm{Rm} * \mathrm{Rm}$ is a quadratic curvature expression, the second sum is over the boundary components, and $\Gamma_{k}$ denotes the orbifold group for each cone. Note the final term is an approximation of the boundary integral, with vanishing error term as $\delta \rightarrow 0$. In terms of Betti numbers,

$$
1-b_{1}\left(U_{j}\right)+b_{2}\left(U_{j}\right)-b_{3}\left(U_{j}\right) \sim \int_{U_{j}} \mathrm{Rm} * \mathrm{Rm}+\sum_{k} \frac{1}{\left|\Gamma_{k}\right|} .
$$

We write $M_{j}=U_{j} \cup V_{j}$, where

$$
U_{j} \cap V_{j} \sim \coprod_{k} S^{3} / \Gamma_{k} .
$$

The Mayer-Vietoris sequence for $U_{j}$ and $V_{j}$ is

$$
0=H_{2}\left(U_{j} \cap V_{j}\right) \rightarrow H_{2}\left(U_{j}\right) \oplus H_{2}\left(V_{j}\right) \rightarrow H_{2}\left(M_{j}\right) \rightarrow H_{1}\left(U_{j} \cap V_{j}\right)=0,
$$

which gives $b_{2}\left(M_{j}\right)=b_{2}\left(U_{j}\right)+b_{2}\left(V_{j}\right)$.

Using (4.2), we can estimate

$$
\sum_{k} \frac{1}{\left|\Gamma_{k}\right|} \leq 1+b_{2}\left(U_{j}\right)+C \cdot \Lambda \leq 1+b_{2}\left(M_{j}\right)+C \cdot \Lambda .
$$

Applying the Gauss-Bonnet formula to the oriented manifold $M_{j}$,

$$
2-2 b_{1}\left(M_{j}\right)+b_{2}\left(M_{j}\right)=\int_{M_{j}} \mathrm{Rm} * \mathrm{Rm}
$$


yields the estimate

$$
b_{2}\left(M_{j}\right) \leq C \cdot \Lambda+2 b_{1}\left(M_{j}\right)-2
$$

so we have

$$
\sum_{k} \frac{1}{\left|\Gamma_{k}\right|} \leq 2 C \cdot \Lambda+2 B_{1}-1 .
$$

The lower volume growth estimate (1.8) clearly implies that the orders of the orbifold groups are bounded strictly from below, so the proposition follows in this case.

In the second case, we use Carron's bound on the number of ends $N$ of a complete space $X$ [Car98, Theorem 0.4]

$$
N \leq 1+C \cdot C_{S} \int_{X} \mid \text { Ric }\left._{-}\right|^{2} d x \leq 1+C \cdot C_{S} \cdot \Lambda,
$$

which, as mentioned above, is also valid for smooth orbifolds. Since we have a volume growth bound (4.1) we can perform a standard bubbling analysis. This analysis was carried out for Einstein metrics in [Ban90a], [Ban90b], see also [Nak92] for a nice description of this bubbling process. The same method works in our case, with a few modifications.

We recall the main steps of the bubbling analysis. Let $S$ denote the singular set of convergence. Note that in contrast to the Einstein case, a point $p \in S$ may actually be a smooth point of the limit. For $0<r_{1}<r_{2}$, we let $D\left(r_{1}, r_{2}\right)$ denote $B\left(p, r_{2}\right) \backslash B\left(p, r_{1}\right)$. Given a singular point $x \in S$, take a sequence $x_{j} \in\left(M_{j}, g_{j}\right)$ such that $\lim _{j \rightarrow \infty} x_{j}=x$ and $B\left(x_{j}, \delta\right)$ converges to $B(x, \delta)$ for all $\delta>0$. We choose a radius $r_{\infty}$ sufficiently small and the sequence $x_{j}$ to satisfy

$$
\sup _{B\left(x_{j}, r_{\infty}\right)}\left|\operatorname{Rm}_{g_{j}}\right|^{2}=\left|R_{g_{j}}\right|^{2}\left(x_{j}\right) \rightarrow \infty \quad \text { as } j \rightarrow \infty,
$$

and

$$
\int_{B\left(x, r_{\infty}\right)}\left|R_{g_{\infty}}\right|^{2} d V_{g_{\infty}} \leq \varepsilon_{0} / 2
$$

where $\varepsilon_{0}$ is the constant in the $\varepsilon$-regularity Theorem 1.2.

We next choose $r_{0}(j)$ so that

$$
\int_{D\left(r_{0}, r_{\infty}\right)}\left|R_{g_{j}}\right|^{2} d V_{g_{j}}=\varepsilon_{0}
$$

and again $D_{j}\left(r_{0}, r_{\infty}\right)=B\left(x_{j}, r_{\infty}\right) \backslash B\left(x_{j}, r_{0}\right)$. An important note, which differs from the Einstein case, the annulus $D\left(r_{0}, r_{\infty}\right)$ may have several components. 
Since the curvature is concentrating at $x, r_{0}(j) \rightarrow 0$ as $j \rightarrow \infty$, the rescaled sequence $\left(M_{j}, r_{0}(j)^{-2} g_{j}, x_{j}\right)$ has a subsequence which converges to a complete, non-compact multi-fold with finitely many singular points, which we denote by

$$
M_{\infty, i_{1}}, 1 \leq i_{1} \leq \#\{S\} .
$$

Note that by assumption, $M_{\infty, i_{i}}$ has bounded Sobolev constant. Since

$$
\int_{D(1, \infty)}|\mathrm{Rm}|^{2} d V_{g} \leq \varepsilon_{0}
$$

there are no singular points outside of $B(x, 1)$.

On the noncompact ends, since we are assuming an upper volume growth estimate, the proof of [TV05a, Theorem 1.3] allows us to conclude that the metric is ALE of order $\tau$ for any $\tau<2$. As in [Ban90a, Proposition 4], we conclude that the neck regions (for large $j$ ) will be arbitrarily close to a portion of a flat cones $\mathbb{R}^{4} / \Gamma$, possibly several cones if $M_{i_{1}}$ has several ends (again in contrast with the Einstein case). The convergence at a singular point $x_{i_{1}}$ is that the ALE multi-fold $M_{\infty, i_{1}}$ is bubbling off, or scaled down to a point in the limit, with each end of $M_{\infty, i_{1}}$ corresponding exactly to a cone at a multi-fold point of the limit $\left(M_{\infty}, g_{\infty}\right)$. An important fact is that the fundamental group of an end and the group of the orbifold cone onto which the end is glued must be isomorphic (together with their actions on $\mathbb{R}^{4}$ ), this also follows from the proof in [Ban90a], see also [Nak92, Theorem 2.5]. In particular, the number of ends of $M_{\infty, i_{1}}$ is the same as the number of components of $\partial B\left(x_{i_{1}}, r_{\infty}\right)$.

To further analyze the degeneration at the singular points, we look at the multifold $M_{\infty, i_{1}}$ with singular set $S_{i_{1}}$. If $S_{i_{1}}$ is empty, then we can stop, as the number of cones must be bounded. If not, we do the same process as above for each singular point of $M_{\infty, i_{1}}$, and obtain ALE multi-folds

$$
M_{\infty, i_{1}, i_{2}}, 1 \leq i_{2} \leq \#\left\{S_{i_{1}}\right\},
$$

each of which are just limits of re-scalings of the original sequence around the appropriate basepoints. If $M_{\infty, i_{1}, i_{2}}$ has singularities, then we repeat the procedure. This process must terminate in finite steps, since in this construction, each singularity takes at least $\varepsilon_{0}$ of curvature: the $L^{2}$-curvature bound (1.6) clearly implies the number of steps in the procedure is bounded by the number $b=\Lambda / \varepsilon_{0}$. As pointed out in [Ban90b], there could be some overlap if any singular point lies on the boundary of $B(1)$ at some stage in the above construction. But there can only be finitely many, and then there must also be a singular point in the interior of $B(1)$, so we still take away at least $\varepsilon_{0}$ of curvature at each step.

Note that in each step of the bubbling process, each end of the multi-fold obtained in the $k$ th step will be glued to one of the cones at a multi-fold singular point of the $(k-1)$ st step, along a neck region which is close to a portion of a flat cone. Again, 
we use the fact that the fundamental group of an end and the group of the orbifold cone onto which the end is glued must be isomorphic (with isomorphic actions on $\mathbb{R}^{4}$ ). Consequently, the bound $N$ on the number of ends (4.3) and the bound $b$ on the number of steps in the process, together imply that the number of cones at any singular point must be bounded by $N^{b}$.

Remark. As was discussed in [TV05b, page 369 ], in the Kähler case only irreducible singular points can occur in limit. We remark that this still holds under the weaker assumptions in this paper. That is, in the Kähler case there are never multiple cones at a singular point of convergence.

Remark. The above proposition was proved using an alternative method in [TV05b, Proposition 7.2]. However, that argument required both a Sobolev constant and a first Betti number bound.

Next, we have

Proposition 4.3. Let $(M, g)$ be a smooth multi-fold with finitely many singular points and $g$ a critical metric. Assume that the number of singular points is uniformly bounded by the number $N_{1}$, that the number of cones at any singular point is uniformly bounded by the number $N_{2}$, and that there exists a constant $V_{0}>0$ so that

$$
\operatorname{Vol}(B(q, s)) \geq V_{0} s^{4}
$$

for all $q \in B(p, 2)$, and $s \leq 2$. If

$$
\|\mathrm{Rm}\|_{L^{2}(B(p, 2))}=\left\{\int_{B(p, 2)}|\mathrm{Rm}|^{2} d V_{g}\right\}^{1 / 2} \leq \varepsilon_{0},
$$

then there exists a constant $A_{0}$ such that

$$
\operatorname{Vol}(B(p, 1)) \leq A_{0},
$$

where $A_{0}$ depends only upon $N_{1}, N_{2}$ and $V_{0}$.

Proof. If $(M, g)$ is smooth, then by Theorem 1.2,

$$
\sup _{B(p, 1)}|\mathrm{Rm}| \leq \frac{1}{4} C \varepsilon_{0} .
$$

By Bishop's volume comparison theorem, we must have $\operatorname{Vol}(B(p, 1)) \leq A^{\prime}$, where $A^{\prime}$ depends only lower volume growth constant $V_{0}$ (since $\varepsilon_{0}$ only depends on $V_{0}$ ).

In the case that $(M, g)$ is a smooth orbifold, we claim that the $\varepsilon$-regularity theorem still holds in this setting. This is because the argument in [TV05a, Theorem 3.1] uses 
integration by parts. One then performs a similar argument, by cutting out small balls of radius $\delta$ around the singular points and verifying that the resulting boundary terms vanish as $\delta \rightarrow 0$ (note that it is crucial that the orbifold points be smooth for this to be valid). Furthermore, Proposition 3.1 is remains valid for a smooth orbifold with a bounded number of singular points. The details, which we omit, are straightforward. Consequently, the estimate (4.4) holds. Next, Bishop's volume comparison theorem remains valid for smooth orbifolds (see for example [Bor93]), with the same constant (or better) as in the smooth case. So for an orbifold (no multiple cone points), we still obtain

$$
\operatorname{Vol}(B(p, 1)) \leq A^{\prime}
$$

In the more general situation of a multi-fold, since there are at most $N_{2}$ cones at each of the $N_{1}$ singular points, clearly we obtain the estimate

$$
\operatorname{Vol}(B(p, 1)) \leq N_{1} N_{2} A^{\prime} \equiv A_{0} .
$$

We also note the following fact, for any metric,

$$
\lim _{r \rightarrow 0} \operatorname{Vol}(B(p, r)) r^{-4}=\omega_{4},
$$

where $\omega_{4}$ is the volume ratio of the Euclidean metric on $\mathbb{R}^{4}$. Clearly, $A_{0} \geq \omega_{4}$.

For any metric $(M, g)$, define the maximal volume ratio as

$$
M V(g)=\max _{x \in M, r \in \mathbb{R}^{+}} \frac{\operatorname{Vol}(B(x, r))}{r^{4}} .
$$

If the theorem is not true, then there exists a sequence of critical manifolds $\left(M_{i}, g_{i}\right)$, with $M V\left(g_{i}\right) \rightarrow \infty$, that is, there exist points $x_{i} \in M_{i}$, and $t_{i} \in \mathbb{R}^{+}$so that

$$
\operatorname{Vol}\left(B\left(x_{i}, t_{i}\right)\right) \cdot t_{i}^{-4} \rightarrow \infty,
$$

as $i \rightarrow \infty$. We choose a subsequence (which for simplicity we continue to denote by the index $i$ ) and radii $r_{i}$ so that

$$
2 \cdot A_{0}=\operatorname{Vol}\left(B\left(x_{i}, r_{i}\right)\right) \cdot r_{i}^{-4}=\max _{r \leq r_{i}} \operatorname{Vol}\left(B\left(x_{i}, r\right)\right) \cdot r^{-4},
$$

We furthermore assume that $x_{i}$ is chosen so that $r_{i}$ is minimal, that is, the smallest radius for which there exists some $p \in M_{i}$ such that $\operatorname{Vol}\left(B_{g_{i}}(p, r)\right) \leq 2 A_{0} r^{4}$, for all $r \leq r_{i}$.

First let us assume that $r_{i}$ has a subsequence converging to zero. For this subsequence (which we continue to index by $i$ ), we consider the rescaled metric $\tilde{g}_{i}=r_{i}^{-2} g_{i}$, so that $B_{g_{i}}\left(x_{i}, r_{i}\right)=B_{\tilde{g}_{i}}\left(x_{i}, 1\right)$. From the choice of $x_{i}$ and $r_{i}$, the metrics $\tilde{g}_{i}$ have bounded volume ratio, in all balls of unit size. 
From the argument above, some subsequence converges on compact subsets to a complete length space $\left(M_{\infty}, g_{\infty}, p_{\infty}\right)$ with finitely many point singularities. The limit could either be compact or non-compact. In either case, the arguments above imply that the limit is a Riemannian multi-fold.

Claim 4.4. The conclusions of Theorem 1.1 hold for the limit $\left(M_{\infty}, g_{\infty}, p_{\infty}\right)$

Proof. In the case that $M_{\infty}$ is compact the claim is trivial. For $M_{\infty}$ non-compact, the remarks at the end of [TV05a, Section 3] shows that assumption (2.7) is satisfied. Also, from [TV05a, Lemma 6.1], the Sobolev constant bound implies a lower volume growth bound (this is also valid for orbifolds), so (2.6) is satisfied in both cases.

If we make the assumption on $b_{1}\left(M_{i}\right)$ (but no Sobolev constant assumption), then the proof is the same as in our previous work [TV05b, Claim 7.1]: since $b_{1}\left(M_{i}\right)<\infty$, the limit must have finitely many bad annuli. The finiteness of ends and upper volume growth estimate then follow from Theorem 2.4. In the case where we assume the Sobolev constant bound (but no $b_{1}$ bound), the result is contained in Theorem 2.5.

From Claim 4.4, we have that $M_{\infty}$ has only finitely many ends, and that there exists a constant $A_{1} \geq 2 A_{0}$ so that

$$
\operatorname{Vol}\left(B_{g_{\infty}}\left(p_{\infty}, r\right)\right) \leq A_{1} r^{4}, \quad \text { for all } r>0 .
$$

If $M_{\infty}$ is compact, then clearly the estimate (4.6) is valid for some constant $A_{1} \geq 2 A_{0}$, since the limit has finite diameter and volume, and the estimate holds for $r \leq 1$.

The inequality

$$
\int_{B_{g_{i}}\left(x_{i}, 2 r_{i}\right)}\left|\mathrm{Rm}_{i}\right|^{2} d V_{i}>\varepsilon_{0},
$$

must hold; otherwise, as remarked above, we would have $\operatorname{Vol}\left(B_{g_{i}}\left(x_{i}, r_{i}\right)\right) \leq A_{0} r_{i}^{4}$, which violates Proposition 4.3.

If the $r_{i}$ are bounded away from zero then there exists a radius $t$ so that

$$
\operatorname{Vol}\left(B_{g_{i}}(p, r)\right) \leq 2 A_{0} r^{4}, \quad \text { for all } r \leq t, p \in M_{i} .
$$

We repeat the argument from the first case, but without any rescaling. Since the maximal volume ratio is bounded on small scales, we can extract an multi-fold limit. The limit can either be compact or non-compact, but the inequality (4.6) will also be satisfied for some $A_{1}$, Following the same argument, we find a sequence of balls satisfying (4.7).

We next return to the (sub)sequence $\left(M_{i}, g_{i}\right)$ and extract another subsequence so that

$$
2600 \cdot A_{1}=\operatorname{Vol}\left(B\left(x_{i}^{\prime}, r_{i}^{\prime}\right)\right) \cdot\left(r_{i}^{\prime}\right)^{-4}=\max _{r \leq r_{i}^{\prime}} \operatorname{Vol}\left(B\left(x_{i}^{\prime}, r\right)\right) \cdot r^{-4}
$$


Again, we assume that $x_{i}^{\prime}$ is chosen so that $r_{i}^{\prime}$ is minimal, that is, the smallest radius for which there exists some $p \in M_{i}$ such that $\operatorname{Vol}\left(B_{g_{i}}(p, r)\right) \leq 2600 A_{1} r^{4}$, for all $r \leq r_{i}$. Clearly, $r_{i}<r_{i}^{\prime}$.

Arguing as above, if $r_{i}^{\prime} \rightarrow 0$ as $i \rightarrow \infty$, then we repeat the rescaled limit construction, but now with scaled metric $\tilde{g}_{i}=\left(r_{i}^{\prime}\right)^{-2} g_{i}$, and basepoint $x_{i}^{\prime}$. We find a limiting multi-fold $\left(M_{\infty}^{\prime}, g_{\infty}^{\prime}, p_{\infty}^{\prime}\right)$, and a constant $A_{2} \geq 2600 A_{1}$ so that

$$
\operatorname{Vol}\left(B_{g_{\infty}^{\prime}}\left(p_{\infty}^{\prime}, r\right)\right) \leq A_{2} r^{4} \quad \text { for all } r>0
$$

For the same reason as above, we must have

$$
\int_{B_{g_{j}}\left(x_{j}^{\prime}, 2 r_{j}^{\prime}\right)}\left|\mathrm{Rm}_{j}\right|^{2} d V_{j}>\varepsilon_{0} .
$$

If $r_{i}^{\prime}$ is bounded below, we argue similarly, but without any rescaling.

We next consider the ratio $r_{i}^{\prime} / r_{i}$. There are 2 possible cases.

Case (i): there exists a subsequence (which we continue to index with $i$ ) satisfying $r_{i}^{\prime}<C r_{i}$ for some constant $C$.

Case (ii):

$$
\lim _{i \rightarrow \infty} \frac{r_{i}^{\prime}}{r_{i}}=\infty
$$

In Case (i) we proceed as follows: We claim that for $i$ sufficiently large, the balls $B\left(x_{i}, 2 r_{i}\right)$ (from the first subsequence) and $B\left(x_{i}^{\prime}, 2 r_{i}^{\prime}\right)$ (from the second) must be disjoint because of the choice in (4.8). To see this, if $B\left(x_{i}, 2 r_{i}\right) \cap B\left(x_{i}^{\prime}, 2 r_{i}^{\prime}\right) \neq \emptyset$, then $B\left(x_{i}^{\prime}, 2 r_{i}^{\prime}\right) \subset B\left(x_{i}, 6 r_{i}^{\prime}\right)$. Then (4.6) and (4.8) yield

$$
\begin{aligned}
2600 A_{1}\left(r_{i}^{\prime}\right)^{4} & =\operatorname{Vol}\left(B\left(x_{i}^{\prime}, r_{i}^{\prime}\right)\right)<\operatorname{Vol}\left(B\left(x_{i}^{\prime}, 2 r_{i}^{\prime}\right)\right) \\
& <\operatorname{Vol}\left(B\left(x_{i}, 6 r_{i}^{\prime}\right)\right) \leq 2 A_{1}\left(6 r_{i}^{\prime}\right)^{4}=2592 A_{1}\left(r_{i}^{\prime}\right)^{4},
\end{aligned}
$$

which is a contradiction (note the last inequality is true for $i$ sufficiently large since (4.6) holds for the limit, which is valid only in Case (i)).

In Case (ii) we argue as follows. If the balls $B\left(x_{i}, 2 r_{i}\right)$ (from the first subsequence) and $B\left(x_{i}^{\prime}, 2 r_{i}^{\prime}\right)$ (from the second) are disjoint for all $i$ sufficiently large, then we proceed to the next step. Otherwise, we look again at the scaling so that $r_{i}^{\prime}=1$ : $\tilde{g}_{i}=\left(r_{i}^{\prime}\right)^{-2} g_{i}$, and basepoint $x_{i}^{\prime}$. Then in this rescaled metric,

$$
\operatorname{Vol}\left(B\left(x_{i}^{\prime}, 1\right)\right)=2600 A_{1}
$$

As above, we have a limiting multi-fold $\left(M_{\infty}^{\prime}, g_{\infty}^{\prime}, p_{\infty}^{\prime}\right)$, satisfying

$$
\operatorname{Vol}\left(B\left(p_{\infty}^{\prime}, 1\right)\right)=2600 A_{1}
$$


Proposition 4.2 implies the number of cones at a multi-fold point is a priori bounded, so from Proposition 4.3, we conclude that

$$
\int_{B_{g_{\infty}^{\prime}}\left(p_{\infty}^{\prime}, 2\right)}|\mathrm{Rm}|^{2} d V>\varepsilon_{0},
$$

There is now a singular point of convergence corresponding to the balls $B\left(x_{i}, r_{i}\right)$ in the first subsequence. But since we are in Case (ii), in the $g_{i}^{\prime}$ metric, these balls must limit to a point in $M_{\infty}^{\prime}$. The only possibility is that the original sequence satisfied

$$
\int_{B_{g_{i}}\left(x_{i}^{\prime}, 2 r_{i}^{\prime}\right)}\left|\mathrm{Rm}_{g_{i}}\right|^{2} d V_{i}>2 \varepsilon_{0}
$$

for all $i$ sufficiently large.

We repeat the above procedure, considering possible Cases (i) and (ii) at each step. At the $k$ th step, we can always account for at least $k \cdot \varepsilon_{0}$ of $L^{2}$-curvature. The process must terminate in finitely many steps from the bound $\left\|\mathrm{Rm}_{i}\right\|_{L^{2}}<\Lambda$. This contradicts (4.5), which finishes the proof.

Remark. In the proof of [TV05b, Theorem 1.2], we neglected to consider the possibility of Case (ii), the above fixes this minor oversight. Another point is that Propositions 4.2 and 4.3 depend crucially on the limiting multi-folds being smooth, which requires the removable singularity result [TV05b, Theorem 6.4]. It will be interesting to find a valid proof not using the removable singularity theorem. Furthermore, it has been proposed long ago that the upper volume growth estimate in Theorem 1.4 should hold without requiring either a Betti number assumption or a Sobolev constant bound (only assuming a lower volume growth bound). However, there is no complete proof of this yet. An extension of the existing techniques will require a highly nontrivial analysis of the connectedness properties of geodesic spheres and annuli. This paper overcomes the difficulty in several important cases, but we emphasize that the result is still not known in full generality.

Theorem 1.4 is proved in a similar manner as the corresponding theorem in [TV05b], using Theorems 1.1, 1.2, and the volume growth estimate in Theorem 1.3.

We next prove Corollary 1.5, which is a simple consequence of the Gauss-Bonnet Theorem and Hirzebruch Signature Theorem in dimension four (see [Bes87]):

$$
\begin{aligned}
8 \pi^{2} \chi(M) & =\frac{1}{6} \int_{M} R^{2}-\frac{1}{2} \int_{M}|\mathrm{Ric}|^{2}+\int_{M}|W|^{2}, \\
12 \pi^{2} \tau(M) & =\int_{M}\left|W^{+}\right|^{2}-\int_{M}\left|W^{-}\right|^{2} .
\end{aligned}
$$


In the anti-self-dual case, $W^{+} \equiv 0$, so we have

$$
\begin{aligned}
8 \pi^{2} \chi(M) & =\frac{1}{6} \int_{M} R^{2}-\frac{1}{2} \int_{M}|\mathrm{Ric}|^{2}+\int_{M}\left|W^{-}\right|^{2}, \\
12 \pi^{2} \tau(M) & =-\int_{M}\left|W^{-}\right|^{2} .
\end{aligned}
$$

Add these equations together to obtain

$$
\begin{aligned}
8 \pi^{2} \chi(M)+12 \pi^{2} \tau(M) & =\frac{R^{2}}{6} \operatorname{Vol}(M)-\frac{1}{2} \int_{M}|\mathrm{Ric}|^{2} \\
& =\frac{R^{2}}{6}-\frac{1}{2} \int_{M}|\operatorname{Ric}|^{2},
\end{aligned}
$$

since the scalar curvature is constant, and $\mathrm{Vol}=1$. The topology of the manifold $M$ is fixed, and the scalar curvature is uniformly bounded, so we find that $\frac{1}{2} \int_{M}|\mathrm{Ric}|^{2}$ is bounded. Also (4.9) yields a bound on $\int_{M}\left|W^{-}\right|^{2}$, so we have the estimate

$$
\int_{M}|\mathrm{Rm}|^{2} d V_{g}<\Lambda
$$

for some constant $\Lambda$. Therefore the assumptions of Theorem 1.4 are satisfied, which finishes the proof.

\section{ALE metrics and removable singularities}

A related problem is to find geometric conditions so that each end of a complete space will be ALE of order $\tau>0$, and to determine the optimal order of decay. In [TV05a] we examined this problem for the following cases:

(a) Self-dual or anti-self-dual metrics with zero scalar curvature.

(b) Scalar-flat metrics with harmonic curvature.

By using Theorems 1.1, 1.2, and the volume growth estimate in Theorem 1.3, we have the following improvement of [TV05a, Theorem 1.3]:

Theorem 5.1. Let $(M, g)$ be a complete, noncompact four-dimensional Riemannian manifold with $g$ of class (a) or (b) satisfying,

$$
\int_{M}\left|\mathrm{Rm}_{g}\right|^{2} d V_{g}<\infty
$$


Vol. 83 (2008)

Assume that

$$
\begin{aligned}
\operatorname{Vol}(B(q, s)) & \geq V_{0} s^{4}, \quad \text { for all } q \in M \text { and } s>0 \\
b_{1}(M) & <\infty
\end{aligned}
$$

then $(M, g)$ has finitely many ends, and each end is ALE of order $\tau$ for any $\tau<2$. If we assume instead that

$$
C_{S}<\infty
$$

then the same conclusion holds.

To conclude, we mention that the following removable singularity theorem for critical metrics is expected:

Let $(M, g)$ be a $C^{0}$-orbifold with singular point at $x$, and $g$ be a critical metric satisfying (1.5). Suppose that

$$
\int_{B(x, 1)}\left|\mathrm{Rm}_{g}\right|^{2} d V_{g}<\infty .
$$

Then the metric $g$ extends to $B(x, 1)$ as a smooth orbifold metric. That is, for some small $\delta>0$, the universal cover of $B(x, \delta) \backslash\{x\}$ is diffeomorphic to a punctured ball $B^{4} \backslash\{0\}$ in $\mathbb{R}^{4}$, and the lift of $g$, after diffeomorphism, extends to a smooth critical metric $\tilde{g}$ on $B^{4}$.

This will be addressed in a forthcoming paper ${ }^{1}$. Such a removable singularity theorem was proved for the special cases of (a),(b), and (c) in [TV05b, Theorem 6.4]. The above generalization is crucial in extending Theorems 1.3, 1.4, and 5.1 to the more general class of critical metrics satisfying (1.5), in particular, Bach-flat metrics.

\section{References}

[Abr85] Uwe Abresch, Lower curvature bounds, Toponogov's theorem, and bounded topology. Ann. Sci. École Norm. Sup. (4) 18 (4) (1985), 651-670. Zbl 0595.53043 MR 0839689

[Abr87] U. Abresch, Lower curvature bounds, Toponogov's theorem, and bounded topology. II. Ann. Sci. École Norm. Sup. (4) 20 (3) (1987), 475-502. Zbl 0651.53031 MR 0925724

[AC91] Michael T. Anderson and Jeff Cheeger, Diffeomorphism finiteness for manifolds with Ricci curvature and $L^{n / 2}$-norm of curvature bounded. Geom. Funct. Anal. 1 (3) (1991), 231-252. Zbl 0764.53026 MR 1118730

\footnotetext{
${ }^{1}$ After completion of this paper, this result was proved by Jeffrey Streets [Str07].
} 
[AG90] Uwe Abresch and Detlef Gromoll, On complete manifolds with nonnegative Ricci curvature. J. Amer. Math. Soc. 3 (2) (1990), 355-374. Zbl 0704.53032 MR 1030656

[And88] Michael T. Anderson, $L^{2}$ harmonic forms on complete Riemannian manifolds. In Geometry and analysis on manifolds (Katata/Kyoto, 1987), Lecture Notes in Math. 1339, Springer-Verlag, Berlin 1988, 1-19. Zbl 0652.53030 MR 0961469

[And89] Michael T. Anderson, Ricci curvature bounds and Einstein metrics on compact manifolds. J. Amer. Math. Soc. 2 (3) (1989), 455-490. Zbl 0999661 MR 0999661

[And05] Michael T. Anderson, Orbifold compactness for spaces of Riemannian metrics and applications. Math. Ann. 331 (4) (2005), 739-778. Zbl 1071.53025 MR 2148795

[Ban90a] Shigetoshi Bando, Bubbling out of Einstein manifolds. Tohoku Math. J. (2) 42 (2) (1990), 205-216. Zbl 0719.53025 MR 1053949

[Ban90b] Shigetoshi Bando, Correction and addition: "Bubbling out of Einstein manifolds". Tohoku Math. J. (2) 42 (4) (1990), 587-588. Zbl 0762.53030 MR 1053949

[BB90] P. Bérard and G. Besson, Number of bound states and estimates on some geometric invariants. J. Funct. Anal. 94 (2) (1990), 375-396. Zbl 0723.53026 MR 1081650

[Bes87] Arthur L. Besse, Einstein manifolds. Springer-Verlag, Berlin 1987. Zbl 0613.53001 MR 0867684

[BKN89] Shigetoshi Bando, Atsushi Kasue, and Hiraku Nakajima, On a construction of coordinates at infinity on manifolds with fast curvature decay and maximal volume growth. Invent. Math. 97 (2) (1989), 313-349. Zbl 0682.53045 MR 1001844

[Bor93] Joseph E. Borzellino, Orbifolds of maximal diameter. Indiana Univ. Math. J. 42 (1) (1993), 37-53. Zbl 0801.53031 MR 1218706

[Car98] Gilles Carron, Une suite exacte en $L^{2}$-cohomologie. Duke Math. J. 95 (2) (1998), 343-372. Zbl 0951.58024 MR 1652017

[Car99] Gilles Carron, $L^{2}$-cohomologie et inégalités de Sobolev. Math. Ann. 314 (4) (1999), 613-639. Zbl 0933.35054 MR 1709104

[CQY07] Alice Chang, Jie Qing, and Paul Yang, On a conformal gap and finiteness theorem for a class of four manifolds. Geom. Funct. Anal. 17 (2) (2007), 404-434. Zbl 1124.53020 MR 2322490

[CT06] Jeff Cheeger and Gang Tian, Curvature and injectivity radius estimates for Einstein 4manifolds. J. Amer. Math. Soc. 19 (2) (2006), 487-525. Zbl 1092.53034 MR 2188134

[GPZ94] Robert E. Greene, Peter Petersen, and Shun-Hui Zhu, Riemannian manifolds of faster-than-quadratic curvature decay. Internat. Math. Res. Notices 1994 (9) (1994), 363-377. Zbl 0833.53037 MR 1301436

[Gro81] Michael Gromov, Curvature, diameter and Betti numbers. Comment. Math. Helv. 56 (2) (1981), 179-195. Zbl 0467.53021 MR 0630949

[Kas88] Atsushi Kasue, A compactification of a manifold with asymptotically nonnegative curvature. Ann. Sci. École Norm. Sup. (4) 21 (4) (1988), 593-622. Zbl 0662.53032 MR 0982335

[Kas89] Atsushi Kasue, A convergence theorem for Riemannian manifolds and some applications. Nagoya Math. J. 114 (1989), 21-51. Zbl 0682.53042 MR 1001487 
[Nak92] Hiraku Nakajima, Convergence theorem of Einstein metrics and ALE spaces. Sūgaku 44 (2) (1992), 133-146. Zbl 0789.53029 MR 1193019

[Str07] Jeffrey Streets, Asymptotic curvature decay and removal of singularities of Bach-flat metrics. Preprint, 2007.arXiv:0708.0869

[Tia90] G. Tian, On Calabi's conjecture for complex surfaces with positive first Chern class. Invent. Math. 101 (1) (1990), 101-172. Zbl 0716.32019 MR 1055713

[TV05a] Gang Tian and Jeff Viaclovsky, Bach-flat asymptotically locally Euclidean metrics. Invent. Math. 160 (2) (2005), 357-415. Zbl 1085.53030 MR 2138071

[TV05b] Gang Tian and Jeff Viaclovsky, Moduli spaces of critical Riemannian metrics in dimension four. Adv. Math. 196 (2) (2005), 346-372. Zbl 02213018 MR 2166311

[Zhu94] Shun-Hui Zhu, A volume comparison theorem for manifolds with asymptotically nonnegative curvature and its applications. Amer. J. Math. 116 (3) (1994), 669-682. Zbl 0953.53027 MR 1277451

Received January 10, 2007

Gang Tian, 702 Fine Hall, Princeton University, Princeton, NJ 08544, U.S.A.

E-mail: tian@math.princeton.edu

Jeff Viaclovsky, Department of Mathematics, University of Wisconsin, Madison, WI 53706,

U.S.A.

E-mail: jeffv@math.wisc.edu 